\title{
Left atrial dysfunction in type 2 diabetes mellitus: insights from cardiac MRI
}

\author{
Bruno Graça • Maria João Ferreira • Paulo Donato • \\ Leonor Gomes • Miguel Castelo-Branco • \\ Filipe Caseiro-Alves
}

Received: 24 March 2014 / Revised: 13 May 2014 / Accepted: 26 June 2014 / Published online: 17 July 2014

(C) European Society of Radiology 2014

\begin{abstract}
Objectives The left atrium (LA) modulates left ventricular filling through reservoir, conduit and booster pump functions. Only limited data exist on LA involvement in type 2 diabetes mellitus (DM2). This study sought to assess LA function in asymptomatic DM2 with cardiac MRI. We hypothesized that cardiac MRI can detect LA dysfunction in asymptomatic DM2.

Methods Forty-five patients with asymptomatic DM2 and 24 normoglycaemic controls were studied. MRI cine imaging was performed to measure LA maximal and minimal volumes. A flow-sensitive phase-contrast gradient-echo sequence was used for flow measurements perpendicular to the orifice of the mitral valve, to quantify active LA stroke volume. LA total, passive and active emptying volumes and fractions were calculated. Results LA reservoir function, namely LA total ejection fraction, was significantly greater in controls compared to patients with DM2 (62.2 \pm 5.2 vs $57.0 \pm 7.6 \%, P=0.004)$. LA passive ejection fraction was also greater in the controls $(26.2 \pm 9.5 \mathrm{vs}$ $16.1 \pm 11.0 \%, P<0.001)$. Regarding parameters of LA booster pump function, LA active ejection fraction was not
\end{abstract}

B. Graça $(\bowtie) \cdot$ M. J. Ferreira • P. Donato • L. Gomes •

M. Castelo-Branco $\cdot$ F. Caseiro-Alves

Faculty of Medicine, University of Coimbra, Azinhaga de Santa

Comba, 3000-548 Coimbra, Portugal

e-mail: brunomgraca@gmail.com

B. Graça $\cdot$ P. Donato · F. Caseiro-Alves

Medical Imaging Department, Coimbra's Hospital Centre and

University, Coimbra, Portugal

\section{J. Ferreira}

Cardiology Department, Coimbra's Hospital Centre and University,

Coimbra, Portugal

L. Gomes

Endocrinology Department, Coimbra's Hospital Centre and

University, Coimbra, Portugal significantly different between groups. DM2 was demonstrated to be an independent determinant of LA function.

Conclusions Cardiac MRI enables the detection of LA dysfunction in asymptomatic DM2, characterized by a reduction in LA reservoir and conduit functions.

Key Points

- Evaluation of left atrial function is feasible with cardiac MRI

- Type 2 diabetes mellitus is associated with left atrial dysfunction

- Left atrial function modulates left ventricular filling

Keywords Cardiac imaging techniques $\cdot$ Magnetic resonance imaging · Transmitral flow $\cdot$ Left atrial function $\cdot$ Diabetes mellitus

$\begin{array}{ll}\text { Abbreviations } \\ \text { DM2 } & \text { type } 2 \text { diabetes mellitus } \\ \text { LA } & \text { left atrium } \\ \text { LAEF } & \text { left atrial ejection fraction } \\ \text { LAmax } & \text { left atrial maximal volume } \\ \text { LAmin } & \text { left atrial minimal volume } \\ \text { LApreA } & \text { left atrial volume pre-atrial contraction } \\ \text { LASV } & \text { left atrial stroke volume } \\ \text { LV } & \text { left ventricle } \\ \text { TMF } & \text { transmitral flow } \\ \text { VENC } & \text { velocity encoded }\end{array}$

\section{Introduction}

Patients with type 2 diabetes (DM2) have an increased risk of developing cardiovascular disease, resulting in significant cardiac morbidity and mortality [1]. In DM2 morphological changes suggestive of heart disease may appear before 
symptoms arise and the prevalence of subclinical left ventricular (LV) dysfunction is increased among these patients [2].

Several mechanisms may underlie LV dysfunction in DM2, including atherosclerosis, microinfarctions, mitochondrial dysfunction, lipotoxicity and accumulation of advanced glycation end products, leading to myocyte hypertrophy, perivascular fibrosis and increased quantities of matrix collagen [3]. All suggested mechanisms leading to LV dysfunction in DM2 include systemic phenomena that can also impact the function of the left atrium (LA).

Left atrial function has been conventionally divided into three phases across the different phases of the cardiac cycle. First, during ventricular systole and isovolumetric relaxation, the LA acts as a reservoir and stores pulmonary venous return. Then, during ventricular diastole, LA emptying consists of two distinct components in subjects with sinus rhythm: the early component is related to passive blood flow from the LA, the pulmonary veins and the LA appendage into the LV; and the late component is related to active LA contraction and is referred to as the LA booster function for LV filling [4]. Through these varying mechanical functions, the LA modulates LV filling and plays a key role in maintaining an optimal cardiac performance.

LA volume and function are robust markers of cardiovascular risk and adverse cardiac outcome across a broad range of cardiovascular pathologies [5-7]. A recent prospective observational follow-up study of DM2 patients without overt cardiovascular disease demonstrated that a dilated LA was a predictor of death and major cardiovascular events [8]. Regarding LA function, its prognostic importance in diabetes has not been evaluated.

Fundamental structural and functional properties of the left atrium are often assessed in the clinical setting using 2D echocardiography.

Cardiac MRI offers several inherent advantages for the assessment of LA size and function owing to its high spatial resolution, superior endocardial border definition, and capacity for unrestricted multiplanar imaging, independent of acoustic windows that may limit echocardiography [9]. Basic measurements of LA size and function can be achieved with cardiac MRI by using the images routinely taken for LV function assessment.

There are limited published data on LA involvement in DM2 $[10,11]$ and, as far as we are aware, there is no study about the role of MRI in the evaluation of LA function in DM2. Accordingly, the purpose of this study was to compare cardiac MRI-derived parameters of LA function between well-controlled uncomplicated DM2 and normoglycaemic control subjects. Moreover, obesity and arterial hypertension are common in the clinical setting of DM2 and could also induce LA dysfunction.

So, we also aim to evaluate how DM2, arterial hypertension and body mass index influence LA function.

We hypothesized that asymptomatic DM2 patients will have reduced LA function compared with normoglycaemic control subjects.

\section{Materials and methods}

Study population

Forty-five patients with DM2 were prospectively recruited from the endocrinology department of our institution. Inclusion criteria included age between 45 and 75 years, no symptoms or history of overt heart disease, no signs or symptoms of cerebrovascular disease, no abnormal findings on routine clinical and physical examination and a normal rest ECG. Patients who had a left ventricular ejection fraction less than $55 \%$, regional LV wall motion abnormalities and valvular heart disease, contraindications to MRI, glomerular filtration rate less than $30 \mathrm{~mL} / \mathrm{min}$, glycated haemoglobin greater than $12 \%$, systolic blood pressure above $160 \mathrm{mmHg}$ and/or diastolic blood pressure above $100 \mathrm{mmHg}$, underlying cardiomyopathy, previous myocardial infarction, coronary revascularization or previous cardiac surgery were excluded. Twenty-four healthy volunteers recruited from the local population served as control and had no history of heart disease, diabetes mellitus or high cholesterol. They had a normal physical examination and ECG. All subjects gave informed consent to participate in the study, which was approved by our institutional ethics committee.

\section{Cardiac MRI protocol}

All images were acquired with electrocardiographic gating, breath-holding and the patient in a supine position. Subjects were imaged on a 3-T MR system (Tim Trio, Siemens, Erlangen, Germany). The basic protocol consisted of cine steady-state free-precession imaging (TR, $3.4 \mathrm{~ms}$; TE, $1.2 \mathrm{~ms}$; in-plane spatial resolution, $1.6 \times 2.1 \mathrm{~mm}$ ) for $\mathrm{LV}$ function and mass. Cine imaging was obtained in 8-12 matching short-axis ( $8 \mathrm{~mm}$ thick with $2 \mathrm{~mm}$ gap) and three standard long-axis planes (two-, three- and four-chamber views). For the calculation of LV mass and function, the endocardial and epicardial borders of the LV myocardium were manually traced on successive short-axis cine images at end-diastole and systole.

A breath-held, retrospectively vector-ECG gated, 2D flowsensitive phase-contrast gradient-echo sequence was used for velocity-encoded (VENC) MRI flow measurements perpendicular to the orifice of the mitral valve. VENC-MRI slices were positioned in early diastole at the tip of the mitral valve leaflets. Typical imaging parameters of VENC-MRI were as follows: slice thickness, $6 \mathrm{~mm}$; repetition time, $4.5 \mathrm{~ms}$; echo time, $2.33 \mathrm{~ms}$; number of segments, 3; acquired temporal resolution, $36 \mathrm{~ms} ; 20^{\circ}$ flip angle, 25 calculated phases and pixel spacing $4.5 \times 3.1 \mathrm{~mm}$. Encoding velocity was set to $130 \mathrm{~cm} / \mathrm{s}[12,13]$. Flow analysis was performed on the through-plane VENC-MRI phase-contrast and magnitude images acquired across the mitral valve. To determine total blood flow and volume, region of interest curves on the VENC-MRI phase-contrast images were drawn in the diastole at the mitral 
valve orifice and propagated to all phases to obtain the transmitral flow (TMF) curve [14] (Fig. 1). From the analysis of the TMF curve, the following measurements were performed: $\mathrm{E}$ and $\mathrm{A}$ mean peak velocities (in centimetres per second), E/A ratio and mitral deceleration time (MDT) (measured from the $\mathrm{E}$ peak to the baseline).

\section{Left atrial measurements}

Left atrial volume measurements were performed in the fourand two-chamber orientations by the biplane area-length methods. In the analysis we excluded the atrial appendage and the pulmonary veins. The parameters of LA size and function included in our analyses were as follows:

- LA minimum volume (LAmin): LA end-diastolic volume at the first frame after mitral valve closure (Fig. 2a, b).

- LA maximum volume (LAmax): LA end-systolic volume right before mitral valve opening (Fig. 2c, d).

\section{LA reservoir function}

- Total LA stroke volume $($ LASV $)=$ LAmax - LAmin.

- Total LA ejection fraction $(\mathrm{LAEF})=$ total LASV/LAmax

\section{LA contractile function}

- Active LASV: directly obtained by VENC-MRI from TMF curves (Fig. 3), using a MATLAB script

- LA volume pre-atrial contraction $($ LApreA $)=$ LAmin + Active LASV

- Active LAEF = active LASV/LApreA

LA conduit function

- Passive LASV = LA max - LApreA

- Passive LAEF = passive LASV/LAmax

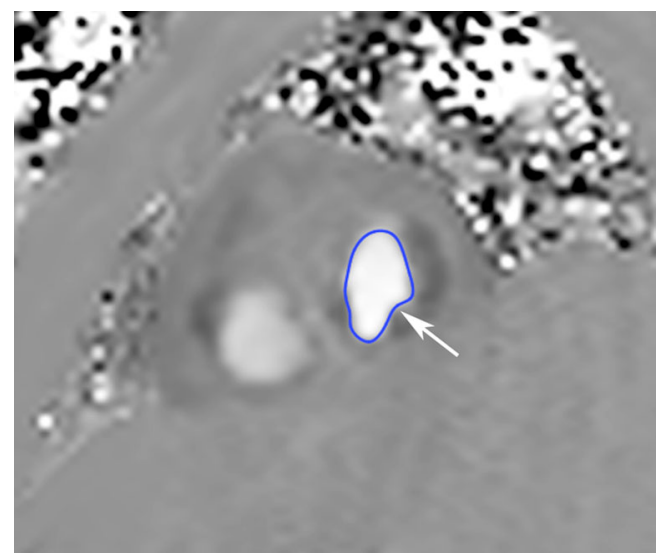

Fig. 1 Phase-encoded MRI image obtained with a phase-contrast sequence shows a region of interest placed at the mitral orifice (arrow). The transmitral flow curve was obtained from this region of interest
MR image analysis

Quantitative image data analysis was performed by using dedicated software (Segment, Medviso, Lund, Sweden) [15]. All functional evaluations were performed within 25 min per patient.

\section{Reproducibility}

In five randomly selected studies from each group, two readers independently measured the LAmax, LAmin and active LASV. One observer remeasured the same 10 studies at a separate time to determine intraobserver agreement from the baseline studies.

Statistical analysis

All continuous variables were tested for normal distribution. All normally distributed data are expressed as means \pm standard deviations. Categorical variables are expressed as counts and percentages. Differences between means of the DM2 group and controls were examined by the unpaired $t$ test. Between-group differences in numbers and percentages were compared using the Chi-squared test.

Univariate and multiple analyses with a forward selection procedure were performed. The goal of these analyses was to determine which factors were responsible for the difference in LA phasic function (total LAEF and passive LAEF) between groups. We employed a two-step strategy for the selection of variables. The first step was that a variable had to be significantly different between groups. If so, in univariate analysis there had to be an association between this variable and the dependent variable with $P<0.1$. The variables fulfilling these criteria were then entered into a multivariable regression analysis and those with $P<0.05$ were considered independently related to the dependent variable. Intraobserver and interobserver variability were assessed by using a Bland-Altman analysis. All computations were performed with software (SPSS, version 20.0; SPSS, Chicago, IL).

\section{Results}

Demographic data and LV parameters of the DM2 group vs. controls are presented in Table 1. Body mass index was significantly increased in the DM2 group $(P<0.001)$. Our population of DM2 had a higher $(53 \%)$ prevalence of systemic arterial hypertension (HT), although not significantly greater than the control group.

All left ventricular volumes and masses were in the normal range, with no significant difference between groups. 
Fig. 2 Cine-MRI slices were acquired in the two-chamber (upper row) and four-chamber (lower row) long-axis orientation. Minimal (a, b) and maximal left atrial $(\mathbf{c}, \mathbf{d})$ volumes were calculated using the biplane arealength method. The atrial appendage and the pulmonary veins were excluded from the measurements
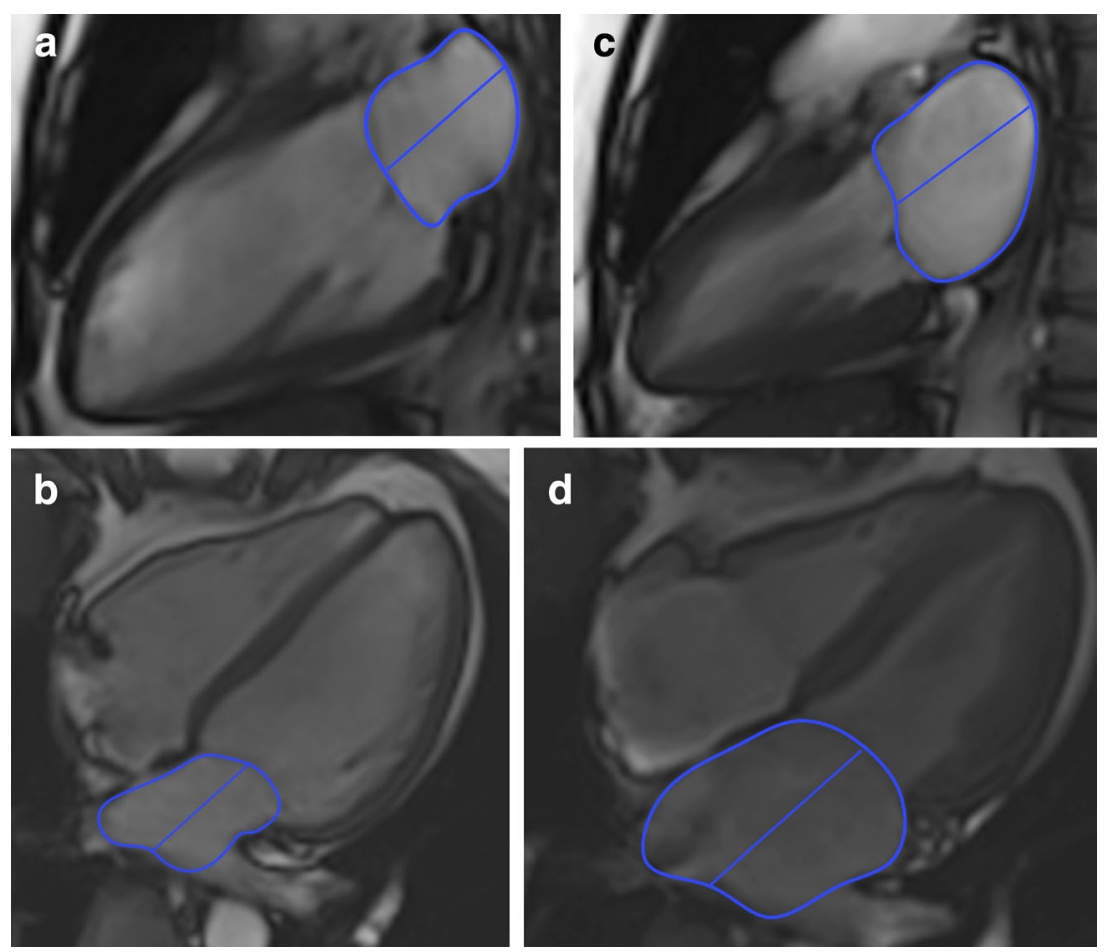

Fig. 3 The transmitral flow curve is composed of two peaks. The first one is the E wave and second one is the A wave. The $\mathrm{E}$ wave corresponds to rapid LV filling at early diastole; the A wave corresponds to late LV filling during end-diastole, secondary to LA contraction. Active left atrial stroke volume was directly obtained from the analysis of the area under the A wave (filled in yellow)

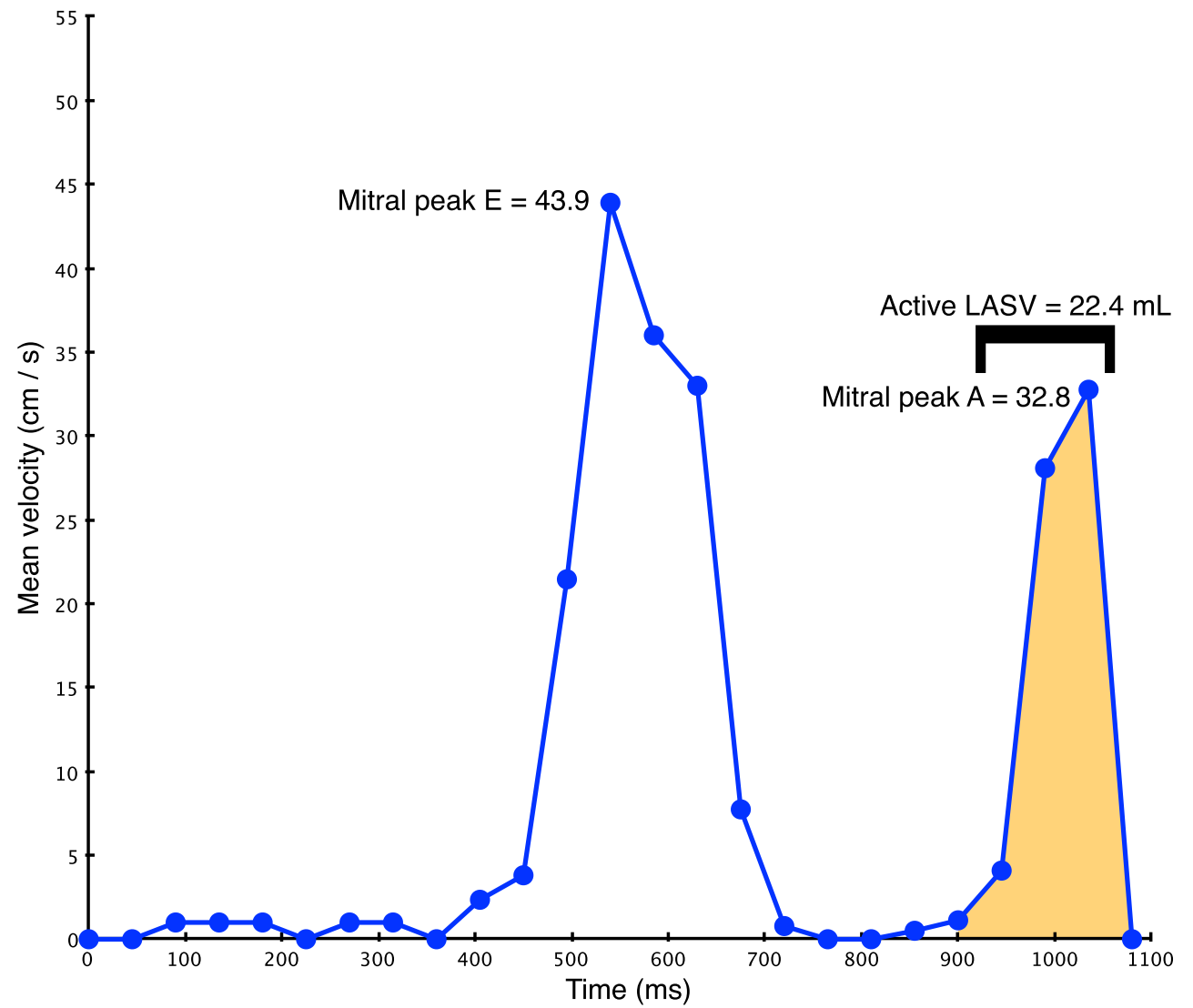


Table 1 Baseline characteristics of the study population and left ventricular measurements

\begin{tabular}{lllr}
\hline & DM2 group $(n=45)$ & Normoglycaemic group $(n=24)$ & $P$ value \\
\hline Age, years & $58.9 \pm 6.9$ & $55.8 \pm 6.8$ & 0.081 \\
Male, $n$ (\%) & $24 / 45(53)$ & $14 / 24(58)$ & 0.801 \\
Body surface area, $\mathrm{m}^{2}$ & $1.87 \pm 0.21$ & $1.83 \pm 0.18$ & 0.374 \\
Body mass index, $\mathrm{kg} / \mathrm{m}^{2}$ & $29.5 \pm 4.4$ & $25.6 \pm 3.1$ & $<0.001$ \\
Hypertension, $n(\%)^{*}$ & $24 / 45(53)$ & $8 / 24(33)$ & 0.011 \\
LV EDV/BSA, $\mathrm{mL} / \mathrm{m}^{2}$ & $77.4 \pm 14.4$ & $79.8 \pm 13.9$ & 0.507 \\
LV EF, \% & $61.6 \pm 7.2$ & $64.5 \pm 5.6$ & 0.119 \\
LV mass / BSA, g/m ${ }^{2}$ & $64.6 \pm 13.1$ & $62.4 \pm 13.4$ & 0.529 \\
Mitral peak E velocity, cm/s & $42.3 \pm 11.9$ & $46.4 \pm 11.6$ & 0.168 \\
Mitral peak A velocity, cm/s & $50.3 \pm 10.7$ & $43.9 \pm 8.9$ & 0.010 \\
Mitral E/A ratio & $0.86 \pm 0.27$ & $1.08 \pm 0.27$ & 0.002 \\
\hline
\end{tabular}

$B P$ blood pressure, $L V$ left ventricle, $E D V$ end-diastolic volume, $B S A$ body surface area, $E F$ ejection fraction

* Blood pressure $>140 / 90 \mathrm{mmHg}$ or treatment with anti-hypertensive medication

Subjects with DM2 had significantly decreased diastolic functional parameters with greater mitral peak A velocity and lower mitral E/A ratio $(P=0.010 ; P=0.002$, respectively).

Intraobserver and interobserver variability of left atrial measurements

The intraobserver limits of agreement were $-2.1 \mathrm{~mL}$ to $0.6 \mathrm{~mL}$ for LAmin, $0.5 \mathrm{~mL}$ to $3.9 \mathrm{~mL}$ for LAmax and $-2.1 \mathrm{~mL}$ to $1.2 \mathrm{~mL}$ for active LASV. The interobserver limits of agreement were $0.02 \mathrm{~mL}$ to $4.2 \mathrm{~mL}$ for LAmin, $-3.0 \mathrm{~mL}$ to $2.6 \mathrm{~mL}$ for LAmax and $-0.9 \mathrm{~mL}$ to $1.2 \mathrm{~mL}$ for active LASV. The corresponding intraclass correlation coefficient values were $0.99,0.99$ and 0.95 for the intraobserver analysis and 0.97 , 0.98 and 0.98 for the interobserver analysis for LAmin, LAmax and active LASV, respectively.

\section{Left atrial measures}

Indexed LA minimum and maximum volumes were not significantly different between groups (Table 2).

A parameter of LA reservoir function, namely total LAEF, was significantly greater in the control group $(P=0.034)$.

A parameter of LA conduit function, namely LA passive ejection fraction, was also significantly greater in the control group $(P<0.001)$.

Regarding parameters of LA booster pump function, including indexed LA active stroke volume and active LAEF, values were not significantly different between groups.

Factors that influence LA function

Table 3 outlines the univariate Pearson correlations for total and passive LAEF. There was no significant correlation between total LAEF and age, body mass index, LV ejection fraction, indexed LV mass, indexed end-diastolic LV volume, mitral peak A velocity and mitral E/A ratio.

There was a significant, low to moderate negative correlation between passive LAEF and age, body mass index and mitral peak A velocity. There was a significant, moderate positive correlation between passive LAEF and mitral E/A ratio. There was no significant correlation between passive LAEF and LV ejection fraction, indexed end-diastolic LV volume and indexed LV mass.

To identify the independent determinants of total and passive LAEF, univariate predictors with $P<0.10$ were all entered into a multiple linear regression model as covariates (Table 4).

On multivariable analysis, total LAEF was independently influenced and reduced in DM2 and with increased BMI. Passive LAEF was related to E/A ratio.

Table 2 Left atrial measurements

\begin{tabular}{lclr}
\hline & $\begin{array}{l}\text { DM2 group } \\
(n=45)\end{array}$ & $\begin{array}{l}\text { Normoglycaemic } \\
\text { group }(n=24)\end{array}$ & $P$ value \\
\hline LAmin/BSA, $\mathrm{mL} / \mathrm{m}^{2}$ & $16.7 \pm 6.9$ & $13.9 \pm 3.9$ & 0.096 \\
LAmax/BSA, $\mathrm{mL} / \mathrm{m}^{2}$ & $37.7 \pm 11.0$ & $36.0 \pm 7.6$ & 0.488 \\
Total LASV/BSA, $\mathrm{mL} / \mathrm{m}^{2}$ & $21.0 \pm 5.8$ & $22.1 \pm 4.4$ & 0.431 \\
Active LASV/BSA, $\mathrm{mL} / \mathrm{m}^{2}$ & $13.8 \pm 3.6$ & $13.2 \pm 3.9$ & 0.522 \\
Passive LASV/BSA, $\mathrm{mL} / \mathrm{m}^{2}$ & $7.2 \pm 5.6$ & $9.0 \pm 5.5$ & 0.243 \\
Total LAEF, \% & $56.9 \pm 8.2$ & $62.2 \pm 9.3$ & 0.034 \\
Passive LAEF, \% & $27.6 \pm 18.2$ & $45.3 \pm 15.5$ & $<0.001$ \\
Active LAEF, \% & $46.7 \pm 11.8$ & $49.0 \pm 12.9$ & 0.513 \\
\hline
\end{tabular}

$B S A$ body surface area, LAmin left atrial minimum volume, LAmax left atrial maximum volume, $L A S V$ left atrial stroke volume, $L A E F$ left atrial ejection fraction 
Table 3 Univariate Pearson correlation coefficients analysis of total LAEF and passive LAEF

\begin{tabular}{|c|c|c|c|c|}
\hline & \multicolumn{2}{|l|}{ Total LAEF } & \multicolumn{2}{|l|}{ Passive LAEF } \\
\hline & Correlation Coefficient & $P$ value & Correlation coefficient & $P$ value \\
\hline Age & 0.20 & 0.876 & -0.30 & 0.017 \\
\hline Body mass index & -0.22 & 0.083 & -0.32 & 0.012 \\
\hline LV EDV/BSA, mL/m² & 0.084 & 0.522 & 0.004 & 0.977 \\
\hline LV mass/BSA, $\mathrm{g} / \mathrm{m}^{2}$ & -0.172 & 0.184 & -0.228 & 0.077 \\
\hline Mitral peak A velocity & 0.17 & 0.190 & -0.29 & 0.024 \\
\hline Mitral E/A ratio & 0.07 & 0.583 & 0.48 & $<0.001$ \\
\hline
\end{tabular}

$L A E F$ left atrial ejection fraction

\section{Discussion}

In this study, MRI-derived parameters of LA function in patients with DM2 were investigated. Differences in LA function were observed between DM2 patients and control subjects. LA phasic function is changed in asymptomatic DM2, with an associated decrease in reservoir (total LAEF) and conduit (passive LAEF) functions. Conversely, there was no change in LA booster function.

We have demonstrated that DM2 and body mass index are independent determinants of LA reservoir function on multivariate analysis. Mitral E/A ratio is an independent determinant of LA passive function.

\section{Left atrial reservoir function}

During LV systole and isovolumic relaxation, the LA functions as a reservoir, receiving blood from the pulmonary veins and storing energy in the form of pressure. This atrial function is mainly modulated by LV contraction, through the descent of the LV base during systole and by LA properties (i.e. relaxation and chamber stiffness) [16].

Table 4 Independent determinants of total LAEF and passive LAEF

\begin{tabular}{lrr}
\hline & $\beta$ & $P$ value \\
\hline Total LAEF & & \\
$\quad$ Diabetes mellitus & -0.15 & 0.017 \\
Arterial hypertension & -0.01 & 0.918 \\
Body mass index & 1.10 & $<0.001$ \\
Passive LAEF & & \\
Diabetes mellitus & -0.18 & 0.182 \\
Hypertension & 0.04 & 0.670 \\
Body mass index & -0.03 & 0.927 \\
Mitral peak A velocity & 0.22 & 0.328 \\
Mitral E/A ratio & 0.89 & $<0.001$ \\
\hline
\end{tabular}

$L A E F$ left atrial ejection fraction
Our group of DM2 patients demonstrated lower total LA ejection fractions compared to normoglycaemic controls. This could by explained by a decrease in LA compliance in the DM2 group [17] in the context of diabetic cardiomyopathy. An impairment of LA compliance in DM2 has been recently demonstrated by Kadappu et al. [11], by showing that echocardiographic parameters of global and segmental strains of the LA were significantly reduced in subjects with DM2.

\section{Left atrial conduit function}

During early LV diastole, the pressure in the left atrium falls and flow in the pulmonary veins increases. During this period, the left atrium acts as a passive conduit (conduit function of the left atrium) and blood is transferred into the LV through the LA via a small pressure gradient and flows passively from the pulmonary veins into the LV. Traditionally the LA passive stroke volume is not easily measured, because while the mitral valve is open, some blood flows directly from the pulmonary veins and LA appendage [16]. In our study, we obtained the LA active atrial emptying volume directly by measurement of transmitral total flow across all the area of the mitral valve, and added this volume to LA minimum volume to establish the LA volume before LA contraction. The LA passive stroke volume was obtained by subtracting this LA volume before LA contraction to LA maximum volume. This method is not confounded by passive diastolic blood flow from the pulmonary veins and LA appendage [18]. Passive LA ejection fraction is the proportion of the LA passive stroke volume to LA maximum volume.

In our study DM2 patients demonstrated lower LA passive ejection fraction compared to normal controls. The explanation for this finding could reside in the LV. Left atrial passive stroke volume is effectively drawn into the left ventricle via LV suction, and it may be more appropriately viewed as a property of LV diastolic function rather than intrinsic LA function. This conduit function is modulated especially by LV diastolic properties (LV relaxation and early diastolic pressures) [19]. 
DM2-related changes in LV diastolic properties are well recognized with a decrease in early diastolic filling, directly influenced by abnormal LV relaxation, and an increased passive stiffness due to remodelling [20]. In fact, the DM2 group of our study showed higher mitral peak A velocity and lower mitral E/A ratio compared to normal controls. Also, mitral E/A ratio was an independent determinant of LA conduit function. Higher mitral peak A velocity and lower mitral E/A ratio are markers of impaired LV relaxation [21], suggesting that there may be a link between LV diastolic properties and LA function.

\section{Left booster pump function}

In the presence of a sinus rhythm, LV filling is completed by atrial contraction. The LA is a contractile chamber that actively empties immediately before the onset of LV systole and establishes final LV end-diastolic volume. LA booster pump function is mostly dependent on intrinsic atrial contractility and becomes increasingly important to the preservation of cardiovascular performance in the presence of reduced LV compliance [16].

In our study, the parameters of LA booster pump function (active LA stroke volume and active LA ejection fraction) were similar in both groups.

Muranaka et al. [10] also showed a reduction in atrial phasic function in diabetes mellitus, as measured by strain rate parameters. Their results also point to an impairment of LA reservoir and conduit functions in patients with DM2 [10]. Asbun and Villarreal [17] demonstrated a relation between diabetic cardiomyopathy and a reduction in LA compliance. A recent study from van Schinkel et al. [22] also demonstrated an association of type 1 diabetes mellitus, aortic stiffness (determined by MRI-assessed pulse wave velocity) and decrease in LA compliance (measured with echocardiographic speckle tracking strain analysis). Kadappu et al. [11] evaluated LA function by strain and strain rate derived from 2D speckle tracking in patients with DM2. Patients with DM2 had altered phasic LA function with impaired LA reservoir, conduit and contractile functions [11].

The findings of our study contribute to the available knowledge, favouring the hypothesis that there is a relationship between DM2, obesity and left atrial dysfunction.

The originality of our study is the use of cardiac MRIderived parameters of LA function.

While MRI represents the current gold standard for assessment of LA size and function [23], there are limited data available on this topic.

Traditionally, LA size and function has been studied by performing 2D echocardiography. However, cyclic changes of LA volume may not be observed directly by $2 \mathrm{D}$ echocardiography because the shape of the LA changes during the heart cycle, and the pattern is influenced by the loading conditions
[24]. Therefore, 2D echocardiography presents only a snapshot view of the LA function.

In contrast, MRI data acquisition is distributed across several cardiac cycles in segmented ECG-gated sequences. Thus, cardiac MRI has been proved to be an effective alternative for accurately assessing the LA volume and phasic function [25].

There is growing evidence that LA size and function serves as an important diagnostic and prognostic factor in a variety of conditions, including DM2 [8]. Therefore, comprehensive evaluation of LA function might be an important clinical factor to stratify the risk of preclinical cardiovascular disease and could be integrated into a routine cardiac MRI protocol for the evaluation of high-risk subjects, including patients with DM2.

There are a few limitations to our study. This study was a case-control study with a relatively small number of subjects and our findings need to be validated in a larger population.

Our findings are limited by an inability to eliminate causal relationships with important clinical factors, such as medications used, serum concentrations of glucose, and glycosylated haemoglobin, dyslipidaemia, retinopathy, microabuminuria and smoking status.

The possibility of influence of myocardial ischaemia or fibrosis on LV or LA function cannot be completely excluded. Although stress imaging is recommended for symptomatic type 2 diabetics, there is still no consensus on the best approach for screening asymptomatic diabetic subjects without known coronary artery disease [26]. In the absence of sufficient clinical indication, it was not deemed ethical to subject these asymptomatic subjects to gadolinium myocardial perfusion and delayed enhancement.

For the analysis of the LA volumes, a true volumetric approach, such as the use of a contiguous short-axis stack, is preferred. However, the biplane area-length method is a validated good compromise between accuracy and speed, as it does not require extra image acquisition apart from that routinely taken for LV function assessment [27].

This study highlights certain key points for the routine use of cardiac MRI to study LA function in diabetic patients. First, evaluation of LA function is feasible with cardiac MRI. Second, body mass index and diabetes independently influence LA function. Third, cardiac MRI shows a decrease in LA reservoir and conduit functions in DM2 and may allow the diagnosis of subclinical LA dysfunction in this high-risk population.

Acknowledgments The scientific guarantor of this publication is Filipe Caseiro-Alves. The authors of this manuscript declare no relationships with any companies whose products or services may be related to the subject matter of the article. This study has received funding under project "DoIT", co-financed by the European Community Fund FEDER through COMPETE - Programa Operacional Factores de Competitividade. Miguel Patrício kindly provided statistical advice for this manuscript. 
Institutional review board approval was obtained. Written informed consent was obtained from all subjects (patients) in this study. Methodology: prospective, case-control study, performed at one institution.

\section{References}

1. Haffner SM, Lehto S, Rönnemaa T et al (1998) Mortality from coronary heart disease in subjects with type 2 diabetes and in nondiabetic subjects with and without prior myocardial infarction. N Engl $\mathrm{J}$ Med 339:229-234

2. Boyer JK, Thanigaraj S, Schechtman KB, Pérez JE (2004) Prevalence of ventricular diastolic dysfunction in asymptomatic, normotensive patients with diabetes mellitus. Am J Cardiol 93: $870-875$

3. Devereux RB, Roman MJ, Paranicas M et al (2000) Impact of diabetes on cardiac structure and function: the strong heart study. Circulation 101:2271-2276

4. Barbier P, Solomon SB, Schiller NB, Glantz SA (1999) Left atrial relaxation and left ventricular systolic function determine left atrial reservoir function. Circulation 100:427-436

5. Tsang MY, Barnes ME, Tsang TS (2012) Left atrial volume: clinical value revisited. Curr Cardiol Rep 14:374-380

6. Viera MJ, Teixeira R, Gonçalves L, Gersh BJ (2014) Left atrial mechanics: echocardiographic assessment and clinical implications. J Am Soc Echocardiogr 27:463-478

7. Hoit BD (2014) Left atrial size and function: role in prognosis. J Am Coll Cardiol 63:493-505

8. Poulsen MK, Dahl JS, Henriksen JE et al (2013) Left atrial volume index: relation to long-term clinical outcome in type 2 diabetes. J Am Coll Cardiol 62:2416-2421

9. Anderson JL, Horne BD, Pennell DJ (2005) Atrial dimensions in health and left ventricular disease using cardiovascular magnetic resonance. J Cardiovasc Magn Reson 7:671-675

10. Muranaka A, Yuda S, Tsuchihashi K et al (2009) Quantitative assessment of left ventricular and left atrial functions by strain rate imaging in diabetic patients with and without hypertension. Echocardiography 26:262-271

11. Kadappu KK, Boyd A, Eshoo S et al (2012) Changes in left atrial volume in diabetes mellitus: more than diastolic dysfunction? Eur Heart J Cardiovasc Imaging 13:1016-1023

12. Caudron J, Fares J, Bauer F, Dacher JN (2011) Evaluation of left ventricular diastolic function with cardiac MR imaging. Radiographics 31:239-259

13. Duarte R, Fernandez G (2010) Assessment of left ventricular diastolic function by MR: why, how and when. Insights Imaging 1:183-192
14. Marsan NA, Westenberg JJ, Ypenburg C et al (2009) Quantification of functional mitral regurgitation by real-time 3D echocardiography: comparison with $3 \mathrm{D}$ velocity-encoded cardiac magnetic resonance. JACC Cardiovasc Imaging 2:1245-1252

15. Heiberg E, Sjögren J, Ugander M et al (2010) Design and validation of segment-freely available software for cardiovascular image analysis. BMC Med Imaging 10:1

16. Rosca M, Lancellotti P, Popescu BA, Piérard LA (2011) Left atrial function: pathophysiology, echocardiographic assessment, and clinical applications. Heart 97:1982-1989

17. Asbun J, Villarreal FJ (2006) The pathogenesis of myocardial fibrosis in the setting of diabetic cardiomyopathy. J Am Coll Cardiol 47:693700

18. Muellerleile K, Groth M, Saring D et al (2012) Evaluation of different magnetic resonance imaging techniques for the assessment of active left atrial emptying. Eur Radiol 22:1904-1911

19. Bowman AW, Kovács SJ (2004) Left atrial conduit volume is generated by deviation from the constant-volume state of the left heart: a combined MRI-echocardiographic study. Am J Physiol Heart Circ Physiol 286:H2416-H2424

20. von Bibra H, St John Sutton M (2010) Diastolic dysfunction in diabetes and the metabolic syndrome: promising potential for diagnosis and prognosis. Diabetologia 53:1033-1045

21. Nagueh SF, Appleton CP, Gillebert TC et al (2009) Recommendations for the evaluation of left ventricular diastolic function by echocardiography. Eur J Echocardiogr 10:165-193

22. Van Schinkel LD, Auger D, van Elderen SG et al (2013) Aortic stiffness is related to left ventricular diastolic function in patients with diabetes mellitus type 1: assessment with MRI and speckle tracking strain analysis. Int J Cardiovasc Imaging 29:633-641

23. Järvinen V, Kupari M, Hekali P, Poutanen VP (1994) Assessment of left atrial volumes and phasic function using cine magnetic resonance imaging in normal subjects. Am J Cardiol 73:1135-1138

24. Cameli M, Lisi M, Righini FM, Mondillo S (2012) Novel echocardiographic techniques to assess left atrial size, anatomy and function. Cardiovase Ultrasound 10:4

25. Hudsmith LE, Petersen SE, Francis JM et al (2005) Normal human left and right ventricular and left atrial dimensions using steady state free precession magnetic resonance imaging. J Cardiovasc Magn Reson 7:775-782

26. Anand DV, Lim E, Hopkins D et al (2006) Risk stratification in uncomplicated type 2 diabetes: prospective evaluation of the combined use of coronary artery calcium imaging and selective myocardial perfusion scintigraphy. Eur Heart J 27:713-721

27. Sievers B, Kirchberg S, Addo M et al (2004) Assessment of left atrial volumes in sinus rhythm and atrial fibrillation using the biplane arealength method and cardiovascular magnetic resonance imaging with TrueFISP. J Cardiovasc Magn Reson 6:855-863 